PROCEEDINGS OF THE

AMERICAN MATHEMATICAL SOCIETY

Volume 131, Number 10, Pages 3231-3239

S 0002-9939(03)07067-9

Article electronically published on March 11, 2003

\title{
LOCATING SUBSETS OF A NORMED SPACE
}

\author{
HAJIME ISHIHARA AND LUMINIŢA VÎŢ \\ (Communicated by Jonathan M. Borwein)
}

\begin{abstract}
Within the framework of Bishop's constructive mathematics, we give conditions under which a bounded convex subset of a uniformly smooth normed space over $\mathbf{R}$ is located, extending results presented recently by $\mathrm{F}$. Richman and H. Ishihara for subsets of a Hilbert space.
\end{abstract}

\section{INTRODUCTION}

It is often pointed out that a constructive study of a mathematical subject provides more information than a classical one, and sometimes produces results about objects that no classical mathematician would ever consider, simply because their existence is taken for granted. Such an example is the notion of a located set, which was first introduced by Bishop [1] in the context of metric spaces, and which can be extended to the more general case of locally convex spaces [4]. A subset $S$ of a metric space $X$ is said to be located if we can compute the distance

$$
\rho(x, S):=\inf \{\rho(x, y): y \in S\}
$$

from any point $x$ of $X$ to $S$. Since this distance is given by an infimum, classically there is no problem with its existence; but constructively the infimum in question is not necessarily computable.

To see how important locatedness is to constructive mathematics, it is enough to mention that results like the Hahn-Banach and separation theorems, or the existence of a projection on a closed subspace of a normed linear space, rely on the locatedness of certain subsets.

For more details on constructive mathematics and located sets, the reader is referred to [1], 2], and [3].

The aim of the present paper is to give conditions under which a bounded convex subset of a uniformly smooth normed space over $\mathbf{R}$ is located, extending the results presented in [8, 6] for subsets of a Hilbert space.

We begin with the fundamental definition.

Definition 1.1. A normed space $E$ is said to be smooth if the limit

$$
\lim _{t \longrightarrow \infty} \frac{\|x+t y\|-\|x\|}{t}
$$

Received by the editors April 19, 2002.

2000 Mathematics Subject Classification. Primary 03F60, 46S30.

Key words and phrases. Functional analysis, constructive mathematics.

The authors were partly supported by a Grant-in-Aid for Scientific Research (C) No. 09640253 of the Ministry of Education, Science, Sports and Culture of Japan.

(C)2003 American Mathematical Society 
exists for all unit vectors $x, y \in E$. The norm of $E$ is said to be Fréchet differentiable if the limit (1.1) is attained uniformly for all unit vectors $y \in E$. The space $E$ is said to be uniformly smooth if the limit (1.1) is approached uniformly for all pairs $(x, y)$ of unit vectors in $E \times E$.

With each vector $x$ in a smooth normed space we associate the normable linear functional $J(x): E \longrightarrow \mathbf{R}$ defined by

$$
(y, J(x))=\|x\| \lim _{t \longrightarrow \infty} \frac{\|x+t y\|-\|x\|}{t} .
$$

A number of properties of this mapping can be found in [9]; for our purposes we only need the following:

- $(x, J(x))=\|J(x)\|^{2}=\|x\|^{2}$.

- $\|y\|^{2} \leq\|x\|^{2}-2(x-y, J(y))$.

\section{An EXAMPLE OF UNIFORMLY SMOOTH NORMED SPACE}

Examples of smooth normed spaces can be found in [7. For uniformly smooth spaces, we have the following proposition.

A normed space $E$ is said to have a uniformly convex dual if for each $\epsilon>0$ there exists $\delta>0$ such that for all normable linear functionals $f$ and $g$ with $\|f\|=\|g\|=$ $1,(u, f+g)>2-\delta$ for some unit vector $u \in E$ implies that $(v, f-g)<\epsilon$ for all unit vectors $v \in E$.

Proposition 2.1. Let $E$ be a separable normed space. Then $E$ is uniformly smooth if and only if $E$ has a uniformly convex dual.

Proof. Suppose that $E$ has a uniformly convex dual. Then since for $t^{\prime} \geq t>0$ we have

$$
\frac{\left\|x-t^{\prime} y\right\|-\|x\|}{-t^{\prime}} \leq \frac{\|x-t y\|-\|x\|}{-t} \leq \frac{\|x+t y\|-\|x\|}{t} \leq \frac{\left\|x+t^{\prime} y\right\|-\|x\|}{t^{\prime}},
$$

it is enough to show that for each $\epsilon>0$ there exists $t>0$ such that for all unit vectors $x, y \in E$,

$$
\frac{\|x+t y\|-\|x\|}{t}-\frac{\|x-t y\|-\|x\|}{-t}<\epsilon
$$

For a given $\epsilon>0$, there exists $\delta>0$ such that for all normable linear functionals $f$ and $g$ with $\|f\|=\|g\|=1$, if $(u, f+g)>2-\delta$ for some unit vector $u \in E$, then $(w, f-g)<\epsilon / 2$ for all unit vectors $w \in E$. Choose $t$ with $t<1 / 2$ and $8 t(2+t)<\delta / 2$. Then for all unit vectors $x, y \in E$, we have $\|x+t y\|>1 / 2$ and $\|x-t y\|>1 / 2$, and hence, letting $u:=(x+t y) /\|x+t y\|$ and $v:=(x-t y) /\|x-t y\|$, we have

$$
\begin{aligned}
\|u-v\| & =\frac{1}{\|x+t y\|\|x-t y\|}\|\| x-t y\|(x+t y)-\| x+t y\|(x-t y)\| \\
& \leq 4(|\|x-t y\|-\|x+t y\||+t(\|x-t y\|+\|x+t y\|)) \\
& \leq 4(2 t+2 t(1+t))=8 t(2+t)<\frac{\delta}{2} .
\end{aligned}
$$

Taking $\gamma:=\min \{t \epsilon /(4(1+t)), \delta / 4\}$, construct normable linear functionals $f$ and $g$ with $\|f\|=\|g\|=1$ such that

$$
(u, f)>1-\gamma \quad \text { and } \quad(v, g)>1-\gamma
$$


by [2, Corollary 7.4.5]. Then we have

$$
\begin{aligned}
(u, f+g) & =(u, f)+(v, g)+(u-v, g) \\
& >2-2 \gamma-\|u-v\| \\
& \geq 2-2 \frac{\delta}{4}-\frac{\delta}{2}=2-\delta .
\end{aligned}
$$

Hence $(w, f-g)<\epsilon / 2$ for all unit vectors $w \in E$; in particular $(y, f-g)<\epsilon / 2$. Since

$$
\|x+t y\|<(x+t y, f)+\|x+t y\| \gamma \leq(x+t y, f)+(1+t) \gamma \leq(x+t y, f)+\frac{t \epsilon}{4}
$$

and similarly

we have

$$
\|x-t y\|<(x-t y, g)+\frac{t \epsilon}{4}
$$

$$
\begin{aligned}
\frac{\|x+t y\|-\|x\|}{t}-\frac{\|x-t y\|-\|x\|}{-t} & =\frac{\|x+t y\|+\|x-t y\|-2}{t} \\
& \leq \frac{(x+t y, f)+(x-t y, g)+t \epsilon / 2-2}{t} \\
& \leq \frac{\epsilon}{2}+(y, f-g)<\epsilon .
\end{aligned}
$$

Conversely, suppose that $E$ is uniformly smooth. Then for each $\epsilon>0$ there exists $\gamma>0$ such that for all unit vectors $x, y \in E$,

$$
0<|t|<\gamma \Longrightarrow\left|\frac{\|x+t y\|-\|x\|}{t}-(y, J(x))\right|<\frac{\epsilon}{4} .
$$

Letting $t:=\gamma / 2$, we have

$$
\begin{aligned}
\|x+t y\| & <\frac{t \epsilon}{4}+t(y, J(x))+1 \\
\|x-t y\| & <\frac{t \epsilon}{4}-t(y, J(x))+1
\end{aligned}
$$

and hence

$$
\|x+t y\|+\|x-t y\|<2+\frac{t \epsilon}{2}
$$

for all unit vectors $x, y \in E$. Let $\delta:=\gamma \epsilon / 4$, let $f$ and $g$ be normable linear functionals with $\|f\|=\|g\|=1$, and assume that $(u, f+g)>2-\delta$ for some unit vector $u \in E$. Then for all unit vectors $v \in E$, we have

$$
\begin{aligned}
2-\frac{t \epsilon}{2} & =2-\delta<(u, f+g) \\
& =(u+t v, f)+(u+t v, g)-t(v, f-g) \\
& \leq\|u+t v\|+\|u-t v\|-t(v, f-g) \\
& <2+\frac{t \epsilon}{2}-t(v, f-g)
\end{aligned}
$$

and hence

$$
(v, f-g)<\epsilon .
$$

Corollary 2.2. For each $p>1$, the space $L_{p}$ is uniformly smooth.

Proof. $L_{p}$ has a uniformly convex dual $L_{q}$ where $1 / p+1 / q=1$; see [2, Corollary 7.3.22, Theorem 7.3.25]. 


\section{The MAIN RESUlts}

Our setting will be a real Banach space $E$.

Lemma 3.1. Let $E$ be a normed space with a Fréchet differentiable norm. Then for each unit vector $u \in E$ and each $\epsilon>0$ there exists $\delta>0$ such that for all unit vectors $v, w \in E$ with $\|v\|=1$,

$$
\|w\|<\delta \Longrightarrow|(v, J(u))-(v, J(u+w))|<\epsilon .
$$

Proof. Let $u \in E$ with $\|u\|=1$ and $\epsilon>0$. Then there exists $\gamma>0$ such that for all unit vectors $v \in E$,

$$
0<|t|<\gamma \Longrightarrow\left|\frac{\|u+t v\|-\|u\|}{t}-(v, J(u))\right|<\frac{\epsilon}{8} .
$$

We may assume that $\gamma<1$. Let $\delta:=\min \{\gamma \epsilon / 32,1 / 2\}$, and suppose that $\|w\|<\delta$. Then letting $x:=(u+w) /\|u+w\|$, we have

$$
0 \leq 1-(u, J(x))=(x-u, J(x)) \leq\|x-u\| \leq \frac{2\|w\|}{\|u+w\|}<4 \delta .
$$

On the other hand, letting $t:=\gamma / 2$, we have

$$
\|u+t v\|+\|u-t v\|<2+\frac{t \epsilon}{4}
$$

for all unit vectors $v \in E$. Therefore for a given unit vector $v \in E$,

$$
\begin{aligned}
1-\frac{t \epsilon}{4} & \leq 1-4 \delta<(u, J(x))=(u, J(u)+J(x))-1 \\
& =(u+t v, J(u))+(u-t v, J(x))-1-t(v, J(u)-J(x)) \\
& \leq\|u+t v\|+\|u-t v\|-1-t(v, J(u)-J(x)) \\
& <\frac{t \epsilon}{4}+1-t(v, J(u)-J(x)),
\end{aligned}
$$

and so

Hence

$$
(v, J(u)-J(x))<\frac{\epsilon}{2}
$$

$$
\begin{aligned}
(v, J(u)-J(u+w)) & =\|u+w\|(v, J(u)-J(x))+(1-\|u+w\|)(v, J(u)) \\
& \leq \frac{3}{2}(v, J(u)-J(x))+\|w\|<\frac{3 \epsilon}{4}+\frac{\epsilon}{32}<\epsilon .
\end{aligned}
$$

Replacing $v$ with $-v$, we have $(v, J(u+w)-J(u))<\epsilon$. Thus

$$
|(v, J(u)-J(u+w))|<\epsilon .
$$

Lemma 3.2. Let $C$ be a bounded located convex subset of a normed space $E$ with a Fréchet differentiable norm, whose diameter is bounded by $M>0$, and let $x \in E$. Then for each $\epsilon>0$ there exists $\tau>0$ such that for all $y \in C$, either

(1) $(y, J(x))+\tau \epsilon / 6<\left(y^{\prime}, J(x)\right)$ for some $y^{\prime} \in C$, or

(2) $(z, J(x))<(y, J(x))+\epsilon$ for all $z \in C$.

Proof. If $\|x\| M<\epsilon$, then for all $z \in C$,

$$
(z-y, J(x)) \leq\|x\|\|z-y\| \leq\|x\| M<\epsilon ;
$$

hence we may assume that $0<\|x\| M$. Let

$$
u:=x /\|x\| \text {. }
$$


Then there exists $\delta>0$ such that for all unit vectors $v, w \in E$ with $\|v\|=1$,

$$
0<|t|<\delta \Longrightarrow\left|\frac{\|u+t v\|-\|u\|}{t}-(v, J(u))\right|<\frac{2 \epsilon}{3\|x\| M}
$$

and

$$
\|w\|<\delta \Longrightarrow|(v, J(u))-(v, J(u+w))|<\frac{2 \epsilon}{3\|x\| M} .
$$

Let $\tau:=\min \{1, \delta\|x\| / M\}$. Then since $\rho(x+y, C)$ exists, either

$$
\left\|x+y-y^{\prime}\right\|^{2}<\|x\|^{2}-\tau \epsilon / 3 \text { for some } y^{\prime} \in C
$$

or

$$
\|x\|^{2}-2 \tau \epsilon / 3<\|x+y-z\|^{2} \text { for all } z \in C .
$$

In the former case, we have

$$
\|x\|^{2} \leq\left\|x+y-y^{\prime}\right\|^{2}-2\left(y-y^{\prime}, J(x)\right)<\|x\|^{2}-\tau \frac{\epsilon}{3}-2\left(y-y^{\prime}, J(x)\right)
$$

and hence

$$
(y, J(x))+\tau \frac{\epsilon}{6}<\left(y^{\prime}, J(x)\right) .
$$

In the latter case, for a given $z \in C$, if $\|x\|\|y-z\|<\epsilon$, then

$$
(z-y, J(x)) \leq\|x\|\|z-y\|<\epsilon ;
$$

hence we may assume that $0<\|x\|\|y-z\|$. Let

$$
\begin{aligned}
v & :=(y-z) /\|y-z\|, \\
w & :=\tau(y-z) /\|x\|, \\
t & :=\tau\|y-z\| /\|x\| .
\end{aligned}
$$

Then $\|w\|=|t|<\delta$. Hence

$$
\begin{aligned}
& \left|\|x\| \frac{\|x+\tau(y-z)\|-\|x\|}{\tau}-(y-z, J(x))\right| \\
= & \|x\|\|y-z\|\left|\frac{\|u+t v\|-\|u\|}{t}-(v, J(u))\right| \\
< & \|x\|\|y-z\| \frac{2 \epsilon}{3\|x\| M} \leq \frac{2 \epsilon}{3}
\end{aligned}
$$

and

$$
\begin{aligned}
& |(y-z, J(x))-(y-z, J(x+\tau(y-z)))| \\
= & \|x\|\|y-z\||(v, J(u))-(v, J(u+w))| \\
< & \|x\|\|y-z\| \frac{2 \epsilon}{3\|x\| M} \leq \frac{2 \epsilon}{3} .
\end{aligned}
$$

Since $C$ is convex and $0 \leq \tau \leq 1$, it follows that $(1-\tau) y+\tau z \in C$; hence

$$
\|x\|^{2}-\tau \frac{2 \epsilon}{3}<\|x+\tau(y-z)\|^{2} .
$$

Therefore we have

$$
\begin{aligned}
0 & <\|x+\tau(y-z)\|^{2}-\|x\|^{2}+2 \tau \epsilon / 3 \\
& =(x, J(x+\tau(y-z)))+\tau(y-z, J(x+\tau(y-z)))-\|x\|^{2}+2 \tau \epsilon / 3 \\
& \leq\|x\|(\|x+\tau(y-z)\|-\|x\|)+\tau(y-z, J(x+\tau(y-z)))+2 \tau \epsilon / 3 .
\end{aligned}
$$


Hence

$$
\begin{aligned}
0 & <\|x\| \frac{\|x+\tau(y-z)\|-\|x\|}{\tau}+(y-z, J(x+\tau(y-z))+2 \epsilon / 3 \\
& \leq((y-z, J(x))+2 \epsilon / 3)+((y-z, J(x))+2 \epsilon / 3)+2 \epsilon / 3,
\end{aligned}
$$

which obviously implies that $(z, J(x))<(y, J(x))+\epsilon$.

Theorem 3.3. Let $C$ be a bounded located convex subset of a normed space $E$ with a Fréchet differentiable norm. Then $\sup \{(z, J(x)): z \in C\}$ exists for each $x \in E$.

Proof. Let $M$ be a bound for the diameter of $C$ and let $x$ be any element of $E$. We will prove that for each $\epsilon>0$ there exists $y \in C$ such that $(z, J(x))<(y, J(x))+\epsilon$ for all $z \in C$. Choose $y_{0} \in C$ and let $\tau$ be as in Lemma 3.2. Construct an increasing binary sequence $\left(\lambda_{n}\right)$ with $\lambda_{0}=0$ and a sequence $\left(y_{n}\right)$ in $C$ such that

$$
\begin{aligned}
& \lambda_{n}=0 \quad \Longrightarrow \quad\left(y_{n-1}, J(x)\right)+\tau \frac{\epsilon}{6}<\left(y_{n}, J(x)\right) \\
& \lambda_{n}=1 \quad \Longrightarrow \quad(z, J(x))<\left(y_{n-1}, J(x)\right)+\epsilon \text { for all } z \in C .
\end{aligned}
$$

Assume we have constructed $\lambda_{0}, \ldots, \lambda_{n}$ and $y_{0}, \ldots, y_{n}$. If $\lambda_{n}=1$, set $\lambda_{n+1}:=1$ and $y_{n+1}:=y_{n}$. If $\lambda_{n}=0$, then by Lemma 3.2 either $\left(y_{n}, J(x)\right)+\tau \frac{\epsilon}{6}<\left(y^{\prime}, J(x)\right)$ for some $y^{\prime} \in C$, or $(z, J(x))<\left(y_{n}, J(x)\right)+\epsilon$ for all $z \in C$. In the former case, set $\lambda_{n+1}=0$ and $y_{n+1}:=y^{\prime}$. In the latter case, set $\lambda_{n+1}=1$ and $y_{n+1}:=y_{n}$. Now choose $N$ such that

$$
M\|x\|<N \tau \frac{\epsilon}{6} .
$$

In order to complete our proof, it will be enough to look at $\lambda_{N}$. If $\lambda_{N}=0$, then

$$
\left(y_{0}, J(x)\right)+N \tau \frac{\epsilon}{6}<\left(y_{N}, J(x)\right),
$$

and hence

$$
N \tau \frac{\epsilon}{6}<\left(y_{N}-y_{0}, J(x)\right) \leq M\|x\|
$$

-a contradiction. Therefore $\lambda_{N}=1$.

Corollary 3.4. Let $C$ be a bounded located convex subset of a uniformly convex Banach space $E$ with a Fréchet differentiable norm. Then $\sup \{(z, f): z \in C\}$ exists for each normable linear functional $f$ on $E$.

Proof. For each normable linear functional $f$ on $E$, there exists $x \in E$ such that $(x, f)=\|f\|^{2}=\|x\|^{2}$ by [2, Proposition 7.3.23]. Hence $J(x)=f$ by the uniqueness of such a normable linear functional; see [5, Lemma 1].

The following lemma will enable us to prove the converse of Theorem 3.3 .

Lemma 3.5. Let $C$ be a bounded convex subset of a uniformly smooth normed space $E$ such that for each $x \in E, \sup \{(z, J(x)): z \in C\}$ exists. Then for each $\epsilon>0$ there exists $\sigma$ with $0<\sigma<1$ such that for every $x \in E$ and $y \in C$, either

(1) $\left\|x-y^{\prime}\right\|<(1-\sigma)\|x-y\|$ for some $y^{\prime} \in C$, or

(2) $\|x-y\|<\|x-z\|+\epsilon$ for all $z \in C$.

Proof. If $\|x-y\|<\epsilon$, then $\|x-y\|<\|x-z\|+\epsilon$ for all $z \in C$. Hence we may assume that $\epsilon / 2<\|x-y\|$. $C$ being bounded, we can choose a positive integer 
$M$ such that $\|x-z\|<M$ for all $z \in C$ and $\epsilon / 4<M$. The space $E$ is uniformly smooth; hence there exists $\delta$ with $0<\delta<2$ such that for all unit vectors $u, v \in E$,

$$
0<|t|<\delta \Longrightarrow\left|\frac{\|u-t v\|-\|u\|}{-t}-(v, J(u))\right|<\frac{\epsilon}{8 M} .
$$

Define $\sigma:=\epsilon \delta /(8 M)$. By our choice of $M$, we have $0<\sigma<1$. Then, since $\sup \{(z, J(x-y)): z \in C\}$ exists, either

$$
(z-y, J(x-y))<\epsilon\|x-y\| \text { for all } z \in C
$$

or

$$
\epsilon\|x-y\| / 2<\left(z_{0}-y, J(x-y)\right) \text { for some } z_{0} \in C .
$$

In the first case, for all $z \in C$ we have

$$
\begin{aligned}
\|x-y\|^{2} & =(x-y, J(x-y))=(x-z+z-y, J(x-y)) \\
& =(x-z, J(x-y))+(z-y, J(x-y)) \\
& <\|x-z\|\|x-y\|+\epsilon\|x-y\|
\end{aligned}
$$

and therefore

$$
\|x-y\|<\|x-z\|+\epsilon .
$$

In the second case, setting $\tau:=\delta\|x-y\| /(2 M)$, we have

$$
0<\tau=\delta \frac{\|x-y\|}{2 M}<\frac{\delta}{2}<1
$$

and hence

$$
y^{\prime}:=y+\tau\left(z_{0}-y\right) \in C .
$$

Note that $\epsilon\|x-y\| / 2<\left(z_{0}-y, J(x-y)\right) \leq\left\|z_{0}-y\right\|\|x-y\|$, and therefore $0<\left\|z_{0}-y\right\|$. Then, letting

$$
\begin{aligned}
u & :=(x-y) /\|x-y\|, \\
v & :=\left(z_{0}-y\right) /\left\|z_{0}-y\right\|, \\
t & :=\tau\left\|z_{0}-y\right\| /\|x-y\|,
\end{aligned}
$$

we have

$$
t=\tau \frac{\left\|z_{0}-y\right\|}{\|x-y\|}=\delta \frac{\left\|z_{0}-y\right\|}{2 M}<\delta,
$$

and

$$
\begin{aligned}
\left\|x-y^{\prime}\right\| & =\left\|x-y-\tau\left(z_{0}-y\right)\right\|=\|x-y\|\|u-t v\| \\
& <\|x-y\|\left(\|u\|-t(v, J(u))+t \frac{\epsilon}{8 M}\right) \\
& =\|x-y\|-\tau \frac{\left(z_{0}-y, J(x-y)\right)}{\|x-y\|}+\tau \frac{\epsilon\left\|z_{0}-y\right\|}{8 M} \\
& <\|x-y\|-\tau \frac{\epsilon}{2}+\tau \frac{\epsilon}{4} \\
& =\|x-y\|-\frac{\delta}{2 M} \frac{\epsilon}{4}\|x-y\| \\
& =(1-\sigma)\|x-y\| .
\end{aligned}
$$


Theorem 3.6. Let $E$ be a uniformly smooth normed space and $C$ a bounded convex subset of $E$. Suppose that $\sup \{(z, J(x)): z \in C\}$ exists for each $x \in E$. Then $C$ is located.

Proof. Let $x$ be an element of $E$ and $\epsilon$ a positive number. We will prove that there exists $y \in C$ such that $\|x-y\|<\|x-z\|+\epsilon$ for all $z \in C$. Let $\sigma$ be the corresponding positive number given by Lemma 3.5 and let $y_{0}$ be an element of $C$. If $\left\|x-y_{0}\right\|<\epsilon$, then $\left\|x-y_{0}\right\|<\|x-z\|+\epsilon$ for all $z \in C$. So we can assume that $\left\|x-y_{0}\right\|>0$. We now construct an increasing binary sequence $\left(\lambda_{n}\right)_{n=0}^{\infty}$ with $\lambda_{0}=0$, and a sequence $\left(y_{n}\right)_{n=0}^{\infty}$ of elements of $C$ such that

$$
\begin{aligned}
& \lambda_{n}=0 \quad \Longrightarrow \quad\left\|x-y_{n}\right\|<(1-\sigma)\left\|x-y_{n-1}\right\|, \\
& \lambda_{n}=1 \quad \Longrightarrow \quad\left\|x-y_{n}\right\|<\|x-z\|+\epsilon \text { for all } z \in C .
\end{aligned}
$$

Suppose we have constructed $\lambda_{0}, \ldots, \lambda_{n}$ and $y_{0}, \ldots, y_{n}$. If $\lambda_{n}=1$, set $\lambda_{n+1}=1$ and $y_{n+1}=y_{n}$. If $\lambda_{n}=0$, then, applying Lemma 3.5, we have either $\left\|x-y^{\prime}\right\|<$ $(1-\sigma)\left\|x-y_{n}\right\|$ for some $y^{\prime} \in C$, or $\left\|x-y_{n}\right\|<\|x-z\|+\epsilon$ for all $z \in C$. In the former case, set $\lambda_{n+1}=0$ and $y_{n+1}=y^{\prime}$; in the latter case, set $\lambda_{n+1}=1$ and $y_{n+1}=y_{n}$. Since $0<\sigma<1$, we have $(1-\sigma)^{n} \longrightarrow 0$ as $n \longrightarrow \infty$. Choose $N$ such that

$$
(1-\sigma)^{n}<\frac{\epsilon}{\left\|x-y_{0}\right\|}
$$

for all $n \geq N$. Now check $\lambda_{0}, \ldots, \lambda_{N}$. If there exists $n \leq N$ such that $\lambda_{n}=1$, then we are done; if $\lambda_{n}=0$ for all $n \leq N$, then

$$
\left\|x-y_{N}\right\|<(1-\sigma)^{N}\left\|x-y_{0}\right\|<\epsilon \leq\|x-z\|+\epsilon
$$

for all $z \in C$.

It is an open question whether we can extend these results to unbounded convex sets.

Corollary 3.7. Let $E$ be a uniformly smooth normed space and $C$ a bounded convex subset of $E$. Then $C$ is located if and only if $\sup \{(z, J(x)): z \in C\}$ exists for all $x \in E$.

Corollary 3.8. Let $E$ be a uniformly convex and uniformly smooth Banach space and $C$ a bounded convex subset of $E$. Then $C$ is located if and only if $\sup \{(z, f)$ : $z \in C\}$ exists for each normable linear functional $f$ on $E$.

Let $T$ be a linear mapping from a normed space $E$ into a normed space $F$, and let $S$ be a mapping from the set $F^{\dagger}$ of all normable linear functionals on $F$ into the set $E^{\dagger}$ of all normable linear functionals on $E$. $T$ and $S$ are called adjoint if

$$
(T x, f)=(x, S f) \quad\left(x \in E, f \in F^{\dagger}\right) .
$$

The mapping $S$ is uniquely determined in terms of $T$, is written $T^{*}$, and is called the adjoint of $T$.

Theorem 3.9. Let $E$ be a normed space, and let $F$ be a uniformly convex and uniformly smooth Banach space. Then a bounded linear mapping $T: E \rightarrow F$ has an adjoint if and only if the image $T(B)$ of the unit ball $B$ is located. 
Proof. Let $C:=T(B)$. Then $C$ is a bounded, convex and balanced subset of $F$. Therefore

$$
\begin{aligned}
C \text { is located } & \Longleftrightarrow \sup \{(y, f): y \in C\} \text { exists for all } f \in F^{\dagger} \\
& \Longleftrightarrow \sup \{|(y, f)|: y \in C\} \text { exists for all } f \in F^{\dagger} \\
& \Longleftrightarrow \sup \{|(T x, f)|: x \in B\} \text { exists for all } f \in F^{\dagger} \\
& \Longleftrightarrow f \circ T \in E^{\dagger} \text { for all } f \in F^{\dagger} \\
& \Longleftrightarrow T^{*} \text { exists. }
\end{aligned}
$$

\section{REFERENCES}

[1] Errett Bishop, Foundations of Constructive Analysis, New York, McGraw Hill, 1967. MR 36:4930

[2] Errett Bishop, Douglas Bridges, Constructive Analysis, Springer-Verlag Berlin Heidelberg, 1985. MR 87d:03172

[3] Douglas Bridges, Fred Richman, Varieties of Constructive Mathematics, Cambridge University Press, 1987. MR 88k:03127

[4] Luminiţa Dediu (Vîţă), The Constructive Theory of Operator Algebras, Ph.D. Thesis, University of Canterbury, New Zealand, 2000.

[5] Hajime Ishihara, On the constructive Hahn-Banach theorem, Bull. London Math. Soc. 21(1989), 79-81. MR 90a:46197

[6] Hajime Ishihara, Locating subsets of a Hilbert space, Proc. Amer. Math. Soc. 129 no. 5 (2001), 1385-1390. MR 2001h:46132

[7] D. Johns and C. Gibson, A constructive approach to the duality theorem for certain Orlicz spaces, Math. Proc. Cambridge Philos. Soc. 89(1981), 49-69. MR 81m:46046

[8] Fred Richman, Adjoints and the image of the ball, Proc. Amer. Math. Soc. 129(2001), 11891193. MR 2001g: 47002

[9] Wataru Takahashi, Nonlinear functional analysis, Yokohama Publishers, 2000. MR 2002k:47001

School of Information Science, Japan Advanced Institute of Science and TechnolOGY, TATSUNOKUChI, ISHIKAWA 923-1292, JAPAN

E-mail address: ishihara@jaist.ac.jp

Department of Mathematics and Statistics, Private Bag 4800, University of CanterBury, New Zealand

E-mail address: luminita@math.net 